\title{
AGILITÉ ET INCLUSION PÉDAGOGIQUE À L'UNIVERSITÉ : UN RETOUR D'EXPÉRIENCE
}

\author{
NATHALIE ORIOL
}

Publié dans Management \& Datascience, le 10 novembre 2020

Catégorie : Application

https://management-datascience.org/articles/14395/

\section{RÉSUMÉ}

La co-modalité (enseignement hybride entre virtuel et présentiel) est un enjeu majeur de l'enseignement universitaire en cette période de crise sanitaire. D'une contrainte appréhendée dans l'urgence, elle peut cependant être source d'agilité et d'inclusion pédagogique pour peu qu'elle se structure autour des modèles de scénarisation d'apprentissage conçus par les sciences de l'éducation. A travers un retour d'expérience d'un jeu sérieux, je propose d'en illustrer les principes fondateurs.

\begin{tabular}{l|l|l|l} 
ETUDE DE CAS & PÉdAGOGIE & TRANSFORMATION DIGITALE & UNIVERSITÉ
\end{tabular}

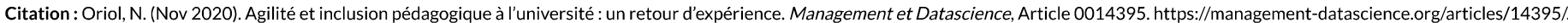

L'auteur:

- Nathalie Oriol

(nathalie.oriol@gredeg.cnrs.fr) - (Pas d'affiliation)

Copyright : @ 2020 I'auteur. Publication sous licence Creative Commons CC BY-ND.

Liens d'intérêts : Le ou les auteurs déclarent ne pas avoir connaissance de conflit d'intérêts impliqués par l'écriture de cet article.

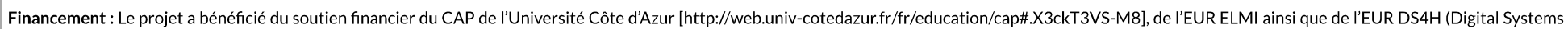
for Humans).

TEXTE COMPLET

Le confinement et la distanciation ont enclenché en marche forcée une vague de flexibilisation pédagogique sans précédent. Mais comment transmettre des connaissances avec justesse lorsque l'on est soudainement contraint de passer à distance ? La principale difficulté à laquelle nous sommes confrontés, dans ce contexte inédit de distanciation, est de conserver sa flexibilité pédagogique et sa capacité à accueillir -dans la mesure du possible- chaque apprenant dans son individualité. Pour autant, faut-il totalement regretter l'idée que soient bousculés ces modes d'enseignements classiques, par nature centrés sur l'enseignant, normés sur un objectif de cours et une évaluation sommative et conçu pour une transmission qui reste majoritairement à sens unique ? Ne peut-on pas imaginer transformer cet impératif à la distanciation en réflexion autour de nouvelles pratiques pédagogiques plus agiles et plus inclusives ? Les fondements de la transformation numérique et des pédagogies dites actives et/ou innovantes à l'université ne datent pas de la crise sanitaire. Elles trouvent racine au sein de nombreux travaux en sciences de l'éducation, dont la mise en application essaime et se concrétise depuis quelques années en France. Je propose, à travers un cas pédagogique, de poser quelques balises d'une conception universelle de l'apprentissage (Hall et al. 2012 [https://www.guilford.com/books/Universal-Design-for-Learning-in-the-Classroom/Hall-Meyer-Rose/9781462506316/editors]) et d'une méthode de scénarisation pédagogique en suivant l'alignement constructif de Biggs et Tang [http://e-journal.uum.edu.my/index.php/mjli/article/view/7624] (2011). Ce cas, intitulé " Crowdfund Your Business ! » (CYB) a été développé au sein de l'Université Côte d'Azur. 


\section{Caractéristiques du cas pédagogique}

Etat des lieux. L'Ecole Universitaire de Recherche ELMI (Economics, Law and Management of Innovation) forme, par le biais d'une trentaine de parcours en Master, des étudiants destinés à occuper des postes de cadre au sein des entreprises et des institutions. Au sein de ces différents parcours, certaines compétences peuvent-être qualifiées de "génériques " c'est-à-dire communes à un grand nombre de formations. C'est le cas de la stratégie financière, de la stratégie digitale et de l'entrepreneuriat. En effet, si ces trois sous-domaines sont abrités sous forme de "majeures » au sein de Master, ils sont d'incontournables fonctions supports (mineures) pour d'autres.

Notre projet pilote «Crowdfund Your Business » est un jeu sérieux en équipe, permettant de déployer une analyse financière et digitale d'une campagne de levée de fonds participatif (crowdfunding). La formation est structurée afin d'être accessible quel que soit le niveau initial des participants dans les domaines considérés. Ainsi, il est dispensé à des étudiants en Masters Economie Gestion, mais également hors de ces champs, à des étudiants de formation scientifique à la recherche de compétences en management de l'innovation. L'objectif du jeu sérieux est de comprendre comment accompagner le financement d'un projet entrepreneurial ou d'une innovation qui ne répondrait pas forcément aux critères du financement bancaire traditionnel (start-up, immatériel...).

Description du projet pédagogique. Le projet se déroule sur 3 séquences d'intervention. La construction par séquence (étape d'apprentissage) diffère de la construction par séance, la séquence regroupant un bloc d'activités associées à des progressions d'acquisition. Chaque séquence s'articule autour de l'acquis d'apprentissage principal du cours et comporte des compétences de savoir-faire mais également de savoir-être intermédiaires.

Ainsi, dans une première phase, les apprenants sont invités à découvrir l'écosystème du financement participatif. En fonctionnant par groupes, ils se voient remettre la documentation d'une campagne réelle pouvant être fondée soit sur du don (love money), soit sur de l'equity (investissement), soit sur du prêt ou sur la base de récompense (reward). L'activité principale est de réaliser un poster afin de convaincre la foule (le reste de la promotion), d'investir dans le projet du groupe. Cela nécessite de maîtriser la communication du projet mais aussi de comprendre le mode de financement. A terme, chaque apprenant dispose d'une somme virtuelle qu'il choisira d'attribuer à un ou plusieurs projets autres que le sien.

Dans une seconde phase, les apprenants doivent se focaliser par groupe sur une campagne de prêt participatif spécifiquement. Ce mode de financement est ici choisi car les risques qui lui sont sous-jacents requièrent une expertise et une éducation financière supérieure aux autres modes. A l'issue de l'étude de cas, ils doivent réaliser un reporting des éléments financiers et extra-financiers qui leur semblent être déterminants dans les choix d'investissement et les expliquer aux autres.

Dans une dernière phase, les groupes formés se voient attribuer un volant de campagnes issues d'une plateforme leader de prêt participatif en Europe. Dotés virtuellement de $1000 €$, ils doivent déterminer comment ventiler cette somme entre les différents projets. Ils doivent enfin présenter leur stratégie et la rentabilité espérée de leur investissement. Le fait d'adopter alternativement le rôle de l'entrepreneur qui lève des fonds et du particulier qui alloue son épargne permet aux apprenants d'appréhender les campagnes sous de multiples dimensions.

\section{L'alignement pédagogique dans le projet CYB}

\section{Fondements méthodologiques du projet CYB}

La conception universelle d'apprentissage offre un canevas pour la création de buts, de méthodes, d'évaluations et de matériel éducatif qui fonctionnent pour tous les individus (Rose et Meyer, 2002 [https://www.cast.org/publications/2002/universal-design-learning-udl-teaching-every-student-rose]). La base de réflexion est l'alignement pédagogique, un principe de base de l'enseignement dans le supérieur. II ne s'agit plus de construire l'enseignement à partir d'objectifs de cours (Biggs et Tang 2011 [http://e-journal.uum.edu.my/index.php/mjli/article/view/7624]) mais à partir d'acquis d'apprentissage centré sur l'étudiant.

Le cas pédagogique a été développé sur la base d'une approche par projet (APP) qui se prête particulièrement au cadre de facilitation de l'insertion professionnelle à un niveau Master. Le principe de l'APP est rendre l'étudiant actif et responsable de ses apprentissages en le mettant en contexte professionnel. Dans cet environnement, l'enseignant tient le rôle de guide. La mise en place d'un environnement structuré et d'une classe flexible, favorisant les interactions, est essentielle. La pédagogie active permet à la fois d'ancrer des techniques professionnelles, mais aussi de développer le savoir être de l'apprenant (communication, rédaction, coordination, persuasion).

Le travail de scénarisation est essentiel et doit être déroulé selon des balises précises. La méthode de l'alignement pédagogique s'appuie sur le backward design (Childre et al. 2009 [https://journals.sagepub.com/doi/abs/10.1177/004005990904100501]) et consiste à formaliser en premier lieu l'acquis d'apprentissage que l'on souhaite proposer, et sur cette base, en déduire la forme de l'évaluation, les outils et les activités que l'on souhaite déployer dans un alignement constructif. Les activités doivent à la fois respecter la progression des compétences envisagées, mais également préparer à l'évaluation. Elles doivent être suffisamment variées pour permettre de correspondre à différentes formes de raisonnement et permettre ainsi à chaque apprenant d'utiliser leurs propres schémas mentaux comme base de travail. Ainsi, l'évaluation devient elle-même formative et inclusive. Celle-ci est ici imaginée en continu et composée principalement de l'appréciation des facilitateurs, mais aussi d'une part apportée par les pairs (exemple : bonus liées aux présentations remportant le plus de suffrages). A l'échelle d'une maquette, l'identification des acquis d'apprentissage intermédiaires et princi- 
paux, formulés à l'aide de la taxonomie de Bloom (1956) [https://www.jstor.org/stable/1170361?seq=1\#metadata_info_tab_contents], permet également de déterminer comment la formation vient nourrir l'approche par compétence globale d'un diplôme.

\section{L'importance des équipements et des systèmes d'information}

Dans le contexte sanitaire actuel, les enseignants doivent moduler leurs modalités d'enseignement parfois en urgence, afin de respecter les mesures prises sur le plan des institutions. Le projet CYB est proposé en mode hybride. II correspond à des heures de cours en présentiel, mais ces dernières peuvent être substituées par des activités en ligne. Le caractère agile et inclusif du projet est étroitement lié à la manière dont les activités sont déployées au sein d'espaces d'apprentissage physiques et virtuels (Oblinger, 2005 [https://er.educause.edu/articles/2005/1/leading-the-transition-from-classrooms-to-learning-spaces]). Le projet est adossé au réaménagement de 2 salles communicantes à l'aide d'un matériel pédagogique adapté aux classes interactives (vidéoprojecteur connecté inter-salle, écrans digitaux, tableaux écritoires mobiles, fauteuils nomades avec tablettes). Cet aménagement a pour objectif de transformer une salle de cours traditionnelle en learning lab (Pour plus de ressources sur la conception de tels espaces d'apprentissage, voir le site collaboratif des espaces d'apprentissage [https://www.learninglab-network.com/author/learninglabnetwork/]).

D'une disposition à l'origine classique, les salles sont à présents équipées d'un matériel permettant une flexibilisation complète des apprentissages, en permettant notamment:

- de créer des espaces de confidentialité entre les groupes afin de gagner en implication dans le jeu

- de prévoir des ateliers de co-construction en groupe simultanés et interchangeables avec plusieurs facilitateurs aux compétences complémentaires

- de bénéficier d'un accès pour les personnes à mobilité réduite avec du matériel spécifique permettant de transformer un fauteuil roulant en poste de travail identique au reste des apprenants.

Les activités peuvent également être proposées en ligne au sein de l'interface Moodle (plateforme d'enseignement en open source) dans un objectif de complémentarité ou de substitution aux activités en présentiel. Ici, le travail de scénarisation est effectué en lien avec l'approche Carpe Diem qui propose une micro-scénarisation des activités pédagogiques sous forme d'E-tivities (activité pédagogiques en ligne efficaces, Salmon 2013 [https://www.routledge.com/E-tivities-The-Key-to-Active-Online-Learning /Salmon/p/book/9780415881760]). Les apprenants peuvent poster leurs réalisations sous forme d'images, de photos, d'infographies, ou cartes mentales en fonction des extensions utilisées. Les votes interactifs et les forums permettent de faire vivre la phase de feedback et d'évaluations par les pairs intégrées à chaque étape d'apprentissage. L'interface permet également d'héberger la diffusion de cours en synchrone via les extensions liées à des softwares de visioconférence, ou de manière asynchrone avec des vidéos sous forme de SPOC (Small Private Online Course). Les apprenants suivent et s'impliquent dans leur progression via l'acquisition de badges qui leur permet de formuler l'acquis de chaque séquence. A titre d'illustration, l'approche de scénarisation peut-être illustrée sur la base de la phase 1 du projet décrite supra (Table 1).

Table 1. Illustration de la conception de la $1^{\text {ère }}$ phase d'apprentissage du projet CYB sur la base du modèle E-tivities (Source : auteur et site de G.Salmon [https://www.gillysalmon.com/e-tivities.html]) 


\begin{tabular}{|c|c|c|}
\hline Eléments & Quoi? & Comment? \\
\hline L'étincelle & Inciter les apprenants & $\begin{array}{l}\text { Fournir un cas réel de } \\
\text { campagne de financement } \\
\text { tout mode confondu à } \\
\text { chaque groupe constitué }\end{array}$ \\
\hline Le but & $\begin{array}{l}\text { Comprendre l'écosystème du } \\
\text { financement participatif et ses } \\
\text { différents modes }\end{array}$ & $\begin{array}{l}\text { Chaque groupe travaillant } \\
\text { sur un mode de financement } \\
\text { précis partagera ensuite la } \\
\text { connaissance acquise avec } \\
\text { les autres }\end{array}$ \\
\hline La tâche & $\begin{array}{l}\text { Collective : monter un visuel } \\
\text { permettant de vendre le projet reçu. } \\
\text { Individuelle : choisir comment } \\
\text { investir } 100 € \text { virtuels parmi les } \\
\text { campagnes }\end{array}$ & $\begin{array}{c}\text { Proposer le projet aux autres } \\
\text { groupes pour les convaincre } \\
\text { Analyser les campagnes de } \\
\text { manière systémique selon la } \\
\text { compréhension de } \\
\text { l'écosystème }\end{array}$ \\
\hline $\begin{array}{l}\text { Réflexion et } \\
\text { feedback }\end{array}$ & $\begin{array}{l}\text { Affiner l'acquis de la séquence } \\
\text { Partager ses connaissances } \\
\text { spécifiques pour la création d'une } \\
\text { connaissance commune }\end{array}$ & $\begin{array}{c}\text { Master class sur } \\
\text { l'écosystème du financement } \\
\text { participatif } \\
\text { Echanges autour des projets } \\
\text { les plus financés }\end{array}$ \\
\hline
\end{tabular}

\section{Conclusion}

La modularité qu'impliquent le mode hybride et l'approche fondée sur l'alignement pédagogique permet ainsi d'obtenir un enseignement agile pouvant s'adapter tant aux grandes qu'aux petites cohortes et se décliner totalement ou partiellement en distanciel/présentiel. Les activités variées et la réflexivité permettent de s'adapter à des publics disposant de peu ou prou de prérequis et à différentes représentations/schémas de pensée. Dans un contexte de crise sanitaire et de pertes de repères des apprenants et des facilitateurs, les sciences de l'éducation sont ainsi un point de repère essentiel afin d'opérer une transition salvatrice de l'enseignement à l'université.

\section{BIBLIOGRAPHIE}

Bloom, B. S. (1956) Taxonomy of Educational Objectives. Vol. 1: Cognitive Domain. Ed.. New York: McKay.

Biggs, J., et Tang, C. (2011). Train-the-trainers: Implementing outcomes-based teaching and learning in Malaysian higher education. Malaysian Journal of Learning and Instruction, vol. 8, 1-19.

Childre A, Sands J.R. et Pope S.T. (2009) Backward Design: Targeting Depth of Understanding for All Learners. TEACHING Exceptional Children. Vol. 41 , n5, p.6-14.

Hall, T., Meyer, A. et Rose, D. (2012). Universal Design for Learning in the Classroom: Practical Applications, Ed. Guilford Press.

Oblinger, D. (2005). Leading the transition from classrooms to learning spaces. Educause quarterly, vol. 1, p.7-12.

Rose, D. H., \& Meyer, A. (2002). Teaching every student in the digital age: Universal design for learning. Ed. Association for Supervision and Curriculum Development. 


\section{CRÉDITS} Le projet a bénéficié du soutien financier du CAP de l'Université Côte d'Azur [http://web.univ-cotedazur.fr/fr/education/cap\#.X3ckT3VS-M8], de l'EUR ELMI ainsi que de
I'EUR DS4H (Digital Systems for Humans).
L'auteur remercie Natalia Timus, et le Centre d'Accompagnement Pédagogique (CAP) de l'Université Côte d'Azur pour leur accompagnement. Le projet a bénéficié du soutien financier du CAP de I'Université Côte d'Azur [http://web.univ-cotedazur.fr/fr/education/cap\#.X3ckT3VS-M8
I'EUR DS4H (Digital Systems for Humans).
L'auteur remercie Natalia Timus, et le Centre d'Accompagnement Pédagogique (CAP) de l'Université Côte d'Azur pour leur accompagnement.

Le projet a bénéficié du soutien financier du CAP de l'Université Côte d'Azur [http://web.univ-cotedazur.fr/fr/education/cap\#.X3ckT3VS-M8]
I'EUR DS4H (Digital Systems for Humans).
L'auteur remercie Natalia Timus, et le Centre d'Accompagnement Pédagogique (CAP) de l'Université Côte d'Azur pour leur accompagnement.

(C) 2020 MANAGEMENT \& DATASCIENCE

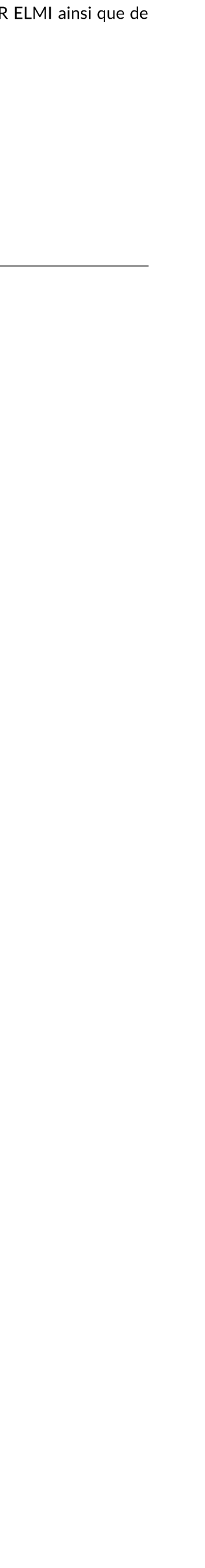

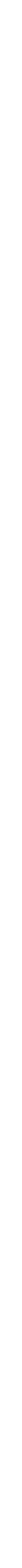

